\title{
Loss of JAK1 Drives Innate Immune Deficiency
}

\author{
Agnieszka Witalisz-Siepracka ${ }^{1}$, Klara Klein ${ }^{1}$, Daniela Prinz ${ }^{1}$, Nicoletta Leidenfrost ${ }^{1}$, \\ Gernot Schabbauer $^{2}$, Alexander Dohnal ${ }^{3}$ and Veronika SexI ${ }^{1 *}$ \\ ${ }^{1}$ Institute of Pharmacology and Toxicology, University of Veterinary Medicine, Vienna, Austria, ${ }^{2}$ Center for Physiology and \\ Pharmacology, Institute for Physiology, Medical University of Vienna, Vienna, Austria, ${ }^{3}$ Tumor Immunology, St. Anna \\ Kinderkrebsforschung, Children's Cancer Research Institute, Vienna, Austria
}

\section{OPEN ACCESS}

Edited by:

Markus G. Seidel,

Medical University of Graz, Austria

Reviewed by:

Lisa Renee Forbes,

Baylor College of Medicine,

United States

Jane Oliaro,

Peter MacCallum Cancer Center,

Australia

Olli Silvennoinen,

University of Helsinki, Finland

*Correspondence:

Veronika Sex

veronika.sex/@vetmeduni.ac.at

Specialty section:

This article was submitted to

Primary Immunodeficiencies,

a section of the journal

Frontiers in Immunology

Received: 28 September 2018 Accepted: 17 December 2018 Published: 08 January 2019

Citation:

Witalisz-Siepracka A, Klein K, Prinz D, Leidenfrost N, Schabbauer G, Dohnal A and SexI V (2019) Loss of JAK1 Drives Innate Immune Deficiency. Front. Immunol. 9:3108. doi: 10.3389/fimmu.2018.03108
The Janus kinase-signal transducers and activators of transcription (JAK-STAT) signaling pathway is critical in tuning immune responses and its dysregulation is tightly associated with cancer and immune disorders. Disruption of interleukin (IL)-15/STAT5 signaling pathway due to the loss of IL-15 receptor chains, JAK3 or STAT5 leads to immune deficiencies with natural killer (NK) cell abnormalities. JAK1, together with JAK3 transmits signals downstream of IL-15, but the exact contribution of JAK1 to NK cell biology remains to be elucidated. To study the consequences of JAK1 deficiency in NK cells, we generated mice with conditional deletion of JAK1 in $\mathrm{NKp}^{+}{ }^{+}$cells (Jak $\left.1^{f / f l} \mathrm{Ncr} 1 \mathrm{Cre}\right)$. We show here that deletion of NK cell-intrinsic JAK1 significantly reduced NK cell numbers in the bone marrow and impaired their development. In line, we observed almost a complete loss of NK cells in the spleen, blood, and liver, proving a crucial role of JAK1 in peripheral NK cells. In line, Jak $1^{\mathrm{fl} /+} \mathrm{Ncr} 1 \mathrm{Cre}$ mice showed significantly impaired NK cell-mediated tumor surveillance. Our data suggest that JAK2 is not able to compensate for the loss of JAK1 in NK cells. Importantly, conditional deletion of JAK2 in $\mathrm{NKp} 46^{+}$cells had no effect on peripheral NK cells revealing that NK cell-intrinsic JAK2 is dispensable for NK cell survival. In summary, we identified that loss of JAK1 in NK cells drives innate immune deficiency, whereas JAK2 deficiency leaves NK cell numbers and maturation unaltered. We thus propose that in contrast to currently used JAK1/JAK2 inhibitors, the use of JAK2-specific inhibitors would be advantageous for the patients by leaving NK cells intact.

\section{Keywords: JAK-STAT, natural killer cells, JAK1, JAK2, tumor surveillance}

\section{INTRODUCTION}

Natural killer (NK) cells are innate lymphocytes which recognize and kill virally infected or transformed cells (1). Deficiency of NK cells is a rare but increasingly appreciated subtype of primary immunodeficiency (PID). Classical NK cell deficiency is characterized by the absence of NK cells in the peripheral blood and results in enhanced susceptibility to viral infections (2).

The Janus kinase (JAK) - signal transducer and activator of transcription (STAT) signaling pathway acts downstream of multiple cytokines, growth factors, and hormones thereby critically regulating immune responses $(3,4)$. Upon binding of a specific ligand to its cognate receptor, conformational changes lead to receptor oligomerization and activation of the receptor-associated JAKs. JAKs auto- and trans-phosphorylate one another and phosphorylate receptor 
chains, providing the docking sites for STAT molecules. STATs then undergo JAK-mediated phosphorylation, dimerize, and translocate to the nucleus, where they regulate the transcription of target genes (5). JAK3 and STAT5 are crucial players in transducing the signal downstream of cytokines which utilize $\gamma c$ receptor (6). "Loss-of-function" (LOF) mutations in genes encoding JAK3 (7) or STAT5B (8) lead to PIDs with an NK cell abnormality underlining the importance of the pathway for innate lymphocytes. The immunodeficiency of these patients has been explained by impaired IL-7 and IL-15 responses (6). Importantly, JAK1 has a dominant role over JAK3 in activating STAT5 downstream of $\gamma$ c-containing cytokine receptors (9). It is attractive to speculate that LOF mutations of JAK1 could also result in PID. To date, only one patient harboring JAK1 germline mutations, where JAK1 was reduced but not absent, has been identified and indeed presented with immune suppression (10).

In mice, complete loss of JAK1 leads to perinatal lethality and newborn mice display a strong reduction of thymocytes and $\mathrm{B}$ cells (11). These observations were confirmed in adult mice: inducible deletion of JAK1 leads to impairment of hematopoietic stem cells (HSCs) homeostasis and markedly reduces the frequencies of $\mathrm{B}$ cells and the $\mathrm{B} 220^{+} \mathrm{CD} 11 \mathrm{c}^{+} \mathrm{NK} 1.1^{+}$ subset of NK cells (12). However, to date, no study has directly analyzed the effect of loss of JAK1 on conventional NK cells.

The first insights into the contribution of JAK1 to NK cell biology derive from studies using JAK inhibitors-approved drugs for treatment of cancers and autoimmune diseases (13). Both, mice and patients treated with the JAK1/JAK2 inhibitor Ruxolitinib showed reduced NK cell numbers, impaired maturation, and function $(14,15)$. Since JAK2 has also been implicated in driving NK cell differentiation $(14,16)$, it remains to be elucidated which of the two kinases is responsible for the observed effects of Ruxolitinib treatment.

Using mice with knockout of Jak1 or Jak2 in $\mathrm{NKp}^{+} 6^{+}$cells, we show here that JAK2 is dispensable for NK cell survival. In contrast, deletion of JAK1 in mature NK cells leads to NK cell deficiency and loss of one allele of Jakl is sufficient to impair tumor growth control. Thus, we identified JAK1 as a key factor for mature NK cells and generated a mouse model of classical NK cell deficiency.

\section{MATERIALS AND METHODS}

\section{Mice and Cell Lines}

$J a k 1^{f l / f l} \quad\left(C 57 B L / 6 N-J a k 1^{t m 1 c(E U C O M M) H m g u / H}\right.$; were kindly provided by Dr. Alexander Dohnal (CCRI, Vienna, Austria). The Jak $1^{\text {tmlc }}$ allele of the mutant was generated from mice with the Jak1 $1^{\text {tmla }}$ knockout first allele (described by International Mouse Phenotyping Consortium https://www.mousephenotype. org) by excision of the lacZ-neo cassette via Flp-recombination.

\footnotetext{
Abbreviations: FACS, fluorescence-activated cell sorting; HSCs, hematopoietic stem cells; ILC, innate lymphoid cells; JAK, Janus kinase; LOF, loss of function; NK, natural killer; PID, primary immunodeficiency; SCID, severe combined immune deficiency; STAT, signal transducer and activator of transcription.
}

The conditional potential of $J a k 1^{f l / f l}$ mice was activated by Cre-recombination and excision of the loxP-flanked exon 3 of Jak1. Tissue-specific recombination was induced by cross breeding of $J a k 1^{f l / f l}$ or $J a k 2^{f l / f l}\left[J a k 2^{t m 1 K u w}\right.$; (17)] with B6N$\operatorname{Tg}\left(\right.$ Ncr1Cre); (18) mice. Stat $5^{f l / f l}$ (19) and Stat $5^{f l / f l}$ Ncr1Cre (18) mice were described before. Jak $1^{f l / f l}$, Ncr1Cre, Stat $5^{f l / f l}$, Stat $5^{f / f l}$ Ncr1Cre mice were on C57B6/N background and $J a k 2^{f l / f l}$ were on mixed background. The experimental animals were age-matched (8-12 weeks) and maintained under specific pathogen-free conditions at the University of Veterinary Medicine, Vienna according to Federation for Laboratory Animal Science Associations (FELASA) guidelines (2014). The animal experiments were approved by the Ethics and Animal Welfare Committee of the University of Veterinary Medicine Vienna and the national authority (Austrian Federal Ministry of Science and Research) according to $\$ \$ 26 \mathrm{ff}$. of Animal Experiments Act, Tierversuchsgesetz 2012-TVG 2012, under licenses BMWF-68.205/0218-II/3b/2012 and BMBWF-68.205/0174-V/3b/2018 and were conducted according to the guidelines of FELASA and ARRIVE. Throughout the paper $J a k 1^{W T}$ refers to pooled data from Jak $1^{f l /+}$ and $J a k 1^{f l / f l}$ mice.

The mouse lymphoma cell lines RMA-Rae1 [kindly provided by Prof. A. Cerwenka; (20)] and YAC-1 were cultured in RPMI1640 (Sigma) complete medium containing 10\% FCS (Bio \& Sell), $100 \mathrm{U} / \mathrm{mL}$ penicillin, $100 \mathrm{mg} / \mathrm{mL}$ streptomycin (Sigma), and $50 \mu \mathrm{M} 2$-mercaptoethanol (Sigma).

\section{In vivo Tumor Model}

$J a k 1^{f l /+}$ and Jak1 $1^{f l /+}$ NcrlCre mice were injected s.c. with $10^{6}$ RMA-Rael cells into both flanks and the tumor growth was monitored every other day. Ten days post injection the mice were sacrificed and the tumor weight was determined. For flow cytometric analysis of tumor infiltrating NK cells, tumors were cut into $\sim 5 \mathrm{~mm}^{2}$ pieces and the single cell suspension was obtained using gentleMACS ${ }^{\mathrm{TM}}$ Octo Dissociator (Miltenyi Biotec) with digestion buffer containing Collagenase D (1 $\mathrm{mg} / \mathrm{mL}$; Sigma Aldrich) and DNAse I (20 mg/mL; Roche).

\section{NK-Cell Isolation, Expansion, and Stimulation}

NK cells were isolated from spleen single-cell suspensions using DX5-labeled MACS beads according to the manufacturer's instructions (Miltenyi Biotec). NK cells were expanded in RPMI1640 complete medium supplemented with 5,000 U/mL rhIL-2 (Proleukin, Novartis) for 7 days. The number of $\mathrm{CD}^{-}{ }^{-} \mathrm{NK} 1.1^{+}$cells was assessed by flow cytometry on day $0,3,5$, and 7 . On day 7 cells were lysed for Western blot analysis. For pSTAT5 analysis $10^{6}$ splenocytes were stimulated with $50 \mathrm{ng} / \mathrm{ml} \mathrm{rmIL-15}$ (PeproTech) for $15 \mathrm{~min}$ and the cells were fixed in $2 \%$ PFA followed by methanol permeabilization and rehydration.

\section{NK-Cell Cytotoxicity Assay}

For in vitro cytotoxicity assays, DX5-MACS-sorted NK cells were expanded for 7 days in IL-2 as described above and mixed at 
indicated effector: target ratios with carboxyfluorescein diacetate succinimidyl ester (CFSE, Molecular Probes, CellTrace CFSE Cell Proliferation Kit) labeled target cells. After $4 \mathrm{~h}$ of incubation at $37^{\circ} \mathrm{C}$, the cells were stained with Sytox Blue Dead Cell Stain (Thermo Fischer) and the specific target cell lysis was assessed by flow cytometry.

\section{Flow Cytometry}

Single cell suspensions were prepared from spleen, bone marrow, or liver. Liver was perfused via the portal vein with $5-10 \mathrm{~mL}$ sterile PBS. Separation of lymphocytes was performed using 37.5\% percoll (GE Healthcare). For blood analysis, the erythrocytes were lysed using BD FACS Lysing
Solution according to manufacturer's protocol (BD Bioscience). The antibodies (clones) targeting following proteins were purchased from eBioscience: CD3 (17A2), CD3e (145-2C11), CD11b (M1/70), CD16/CD32 (93), CD19 (eBio1D3) CD27 (LG.7F9), CD49b (DX5), CD122 (5H4), CD226 (10E5), Gr-1 (RB6-8C5), KLRG1 (2F1), Ly49A (A1), Ly49G2 (eBio4D11), NKG2A/C/E (20d5), NKG2D (CX5), NKp46 (29A1.4), NK1.1 (PK136), and Ter119 (TER-119). CD49a (Ha31/8) and pSTAT5 [47/Stat5(pY694)] antibodies were purchased from BD Pharminogen and pan-Rael (186107) was purchased from R\&D Systems. Total cell numbers were assessed by flow cytometry using counting beads Count Bright Beads (Invitrogen). Flow cytometry experiments were performed
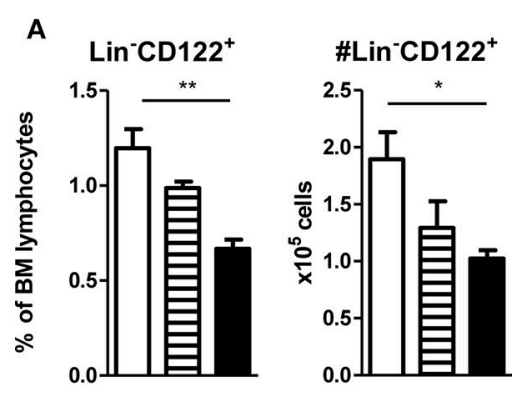

C

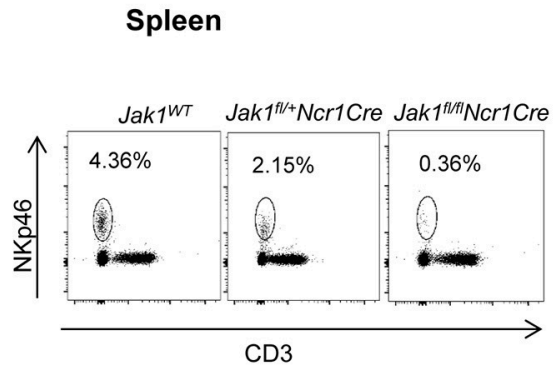

E

Blood

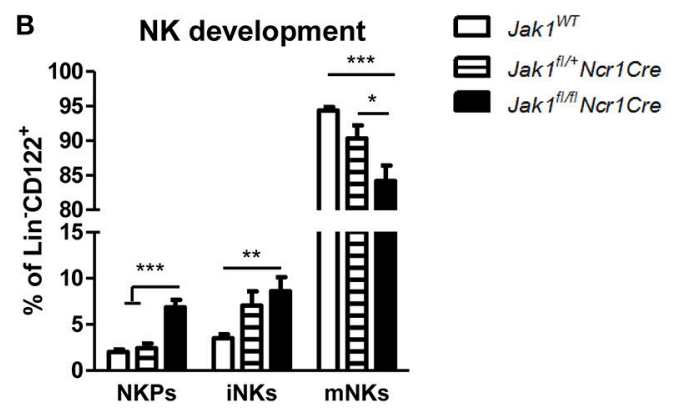

\section{D}

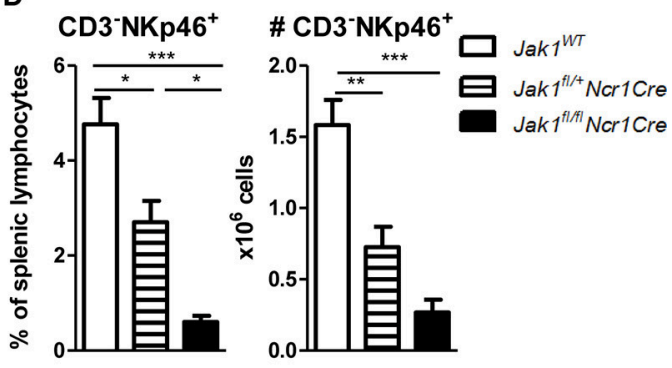

F Liver
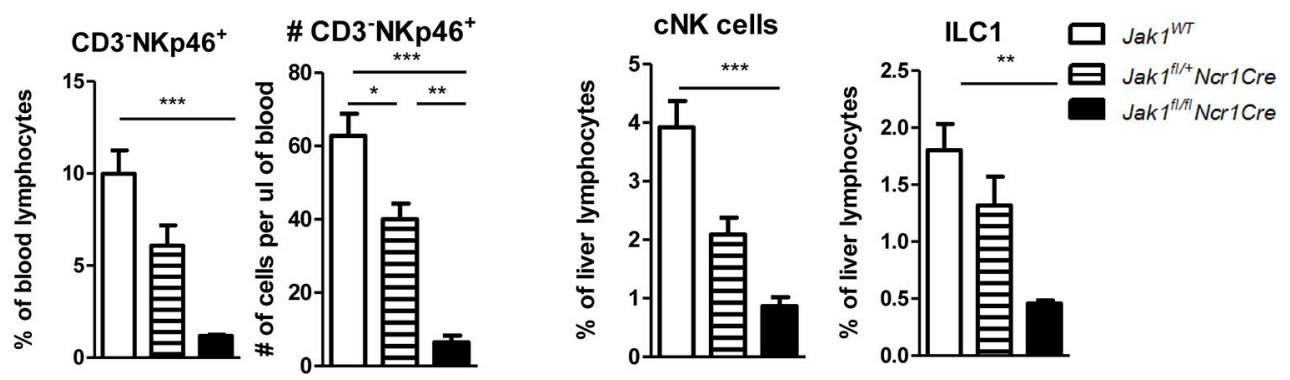

FIGURE 1 | Loss of JAK1 in NKp46+ cells leads to an almost complete absence of peripheral NK cells. (A) Frequency (left panel) and total numbers (right panel) of $\mathrm{Lin}^{-}\left(\mathrm{CD} 3^{-} \mathrm{CD} 19^{-} \mathrm{Ly}^{-6 G^{-}}{ }^{-}\right.$Ter119- $\left.{ }^{-}\right) \mathrm{CD}_{122^{+}} \mathrm{NK}$ cells in the bone marrow were assessed by flow cytometry. (B) Bone marrow $\mathrm{Lin}^{-} \mathrm{CD} 122^{+}$cells were further divided into NK precursors (NKPs: NKp46-NK1.1-), immature NK cells (iNKs: NKp46-NK1.1 ${ }^{+}$), and mature NK cells (mNKs: NKp46 $6^{+} \mathrm{NK1.1^{+ }}$ ). (C) Frequency of $\mathrm{CD}^{-}{ }^{-} \mathrm{NKp} 46^{+}$NK cells in the spleen was assessed by flow cytometry and representative plots are shown. (D,E) Frequency (left panel) and total numbers (right panel) of $\mathrm{CD}^{-}{ }^{-} \mathrm{NKp} 46^{+} \mathrm{NK}$ cells in the (D) spleen and (E) blood were assessed by flow cytometry. (F) Frequency of conventional NK cells $\left(\mathrm{CD} 3^{-} \mathrm{NK} 1.1^{+} \mathrm{NKp} 46^{+} \mathrm{CD} 49 b^{+}\right.$, left panel) and ILC1 cells (CD3 ${ }^{-} \mathrm{NK} 1.1^{+} \mathrm{NKp}^{+} 6^{+} \mathrm{CD} 49 a^{+}$, right panel) was analyzed in the liver of Jak $1^{W T}$, Jak $1^{f l /}+$ Ncr1Cre, and Jak ${ }^{f l / f l}$ Ncr1Cre mice by flow cytometry. (A,B,D-F) Bar graphs represent mean \pm SEM of $1-2$ independent experiments; $n=3-11 .{ }^{\star} p<0.05,{ }^{* \star} p<0.01,{ }^{\star \star *} p<0.001$. 

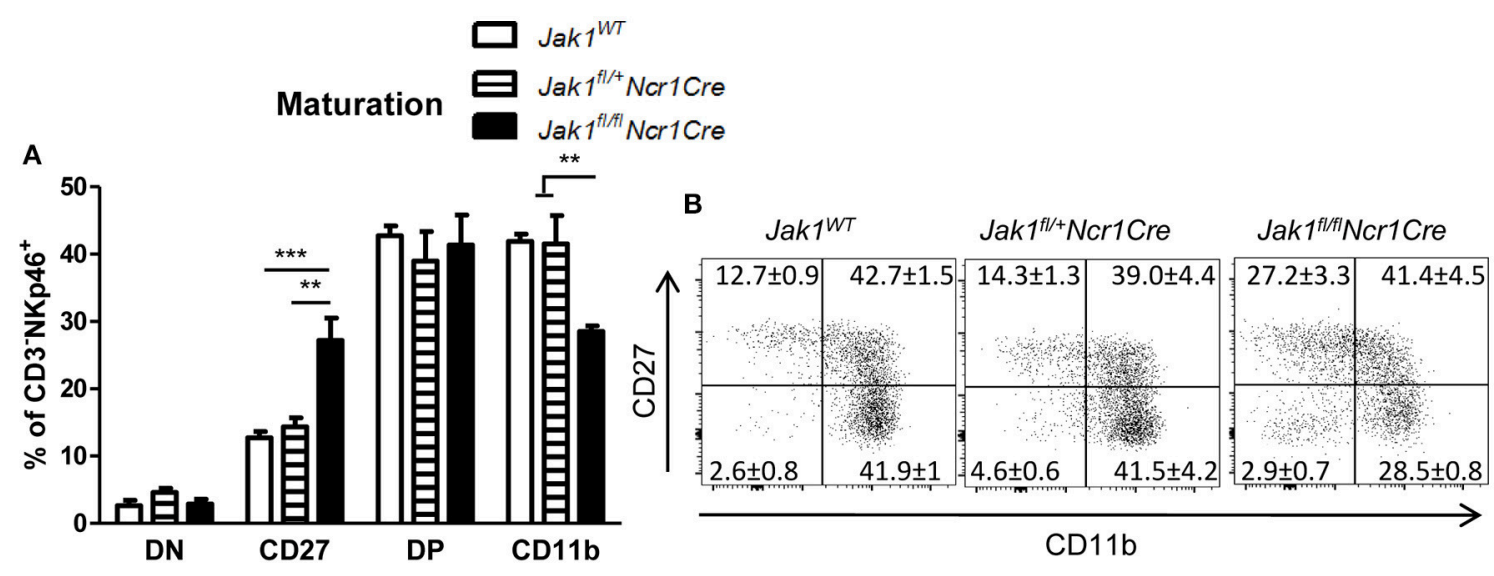

C

pSTAT $5^{+}$after IL-15

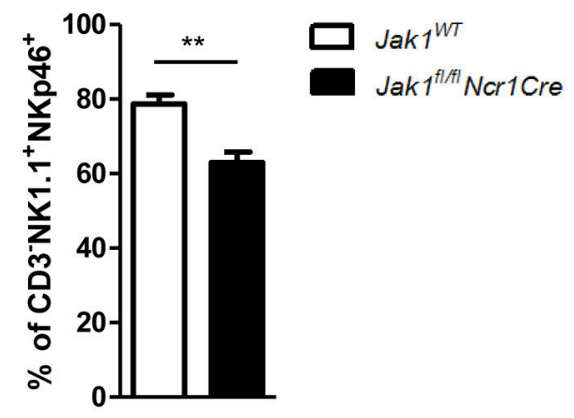

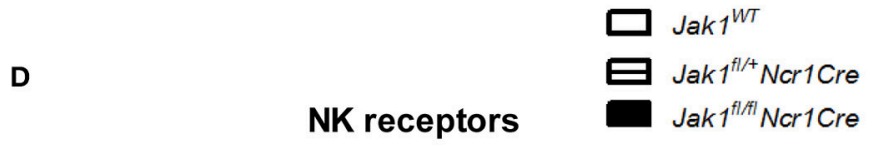

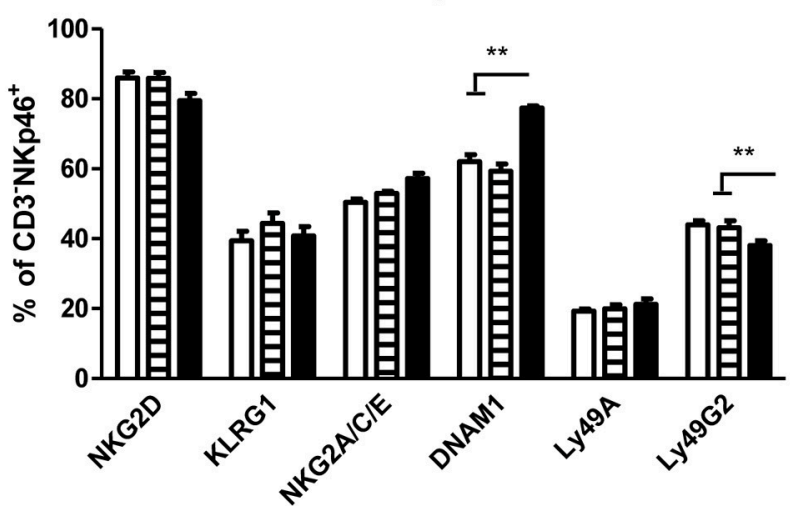

FIGURE 2 | The remaining Jak $1^{f l / f l}$ Ncr1Cre NK cells show an immature phenotype. (A,B) Splenic CD3- NKp46 ${ }^{+}$NK cells were analyzed for expression of CD27 and

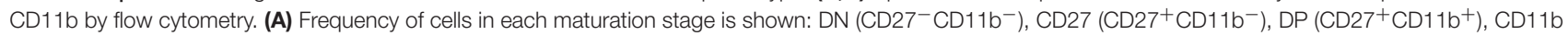
$\left(\mathrm{CD} 27^{-} \mathrm{CD}_{11 \mathrm{~b}^{+}}\right)$. The total numbers of cells in each maturation stage are shown in Figure S1C. (B) Representative plots are shown. (C) pSTAT5(Y694) $)^{+}$cells were analyzed within the $\mathrm{CD}^{-} \mathrm{NKp} 46^{+} \mathrm{NK} 1.1^{+}$population ex vivo after 15 min stimulation with IL-15 by flow cytometry. (D) Splenic CD3- NKp46 ${ }^{+}$NK cells were analyzed for expression of the indicated activating and inhibitory receptors by flow cytometry. The percentage of NK cells positive for each receptor is shown. The median fluorescence intensity data is presented in Figure S1D. (A-D) Bar graphs and numbers on the plots represent mean \pm SEM of 2 independent experiments; $n=5-8$. ${ }^{* *} p<0.01,{ }^{* * *} p<0.001$.

on a BD FACSCanto II (BD Bioscience) or Cytoflex (Beckman Coulter) and analyzed using BD FACSDiva V8.0 (BD Bioscience), CytExpert (Beckman Coulter) or FlowJo V10 (FlowJo, LLC) software.

\section{Western Blot}

Cell lysis, SDS-PAGE, and Western blots were performed as described previously (21). The detection of chemiluminescence was performed using Clarity Western ECL substrate (BioRad) and the ChemiDocT XRS+ Molecular Imager (BioRad) and analyzed by Image Lab software (BioRad). The following antibodies were used: anti- $\beta$-actin (C4, sc-47778) from Santa Cruz as loading control, anti-JAK2 (D2E12; \#3230), antiPerforin (\#3693) and anti-JAK1 (\#3332) from Cell Signaling Technology.

\section{Statistical Analysis}

Unpaired $t$-tests or one-way ANOVA with Tukey post tests were performed using GraphPad Prism version 5.00 (GraphPad Software). The level of significance is indicated for each experiment $\left({ }^{*} p<0.05 ;{ }^{* *} p<0.01 ;{ }^{* * *} p<0.001\right)$.

\section{RESULTS}

\section{JAK1 Deletion Reduces NK Cell and ILC1 Numbers in a Dose-Dependent Manner}

The JAK1/JAK2 inhibitor Ruxolitinib has been shown to reduce NK cell numbers, maturation, and function $(14,15)$. To compare and address the contribution of JAK1 and JAK2 for NK cell biology we generated mice with conditional deletion of either JAK1 or JAK2 in NKp $46^{+}$cells. We thus crossed Ncr1Cre (18) 
mice with $J a k 1^{f l / f l}$ or $J a k 2^{f l / f l}$ [see Materials and Methods section and (17)] mice, respectively. NK cells develop in the bone marrow from NK cell precursors (NKPs), which are defined as $\mathrm{Lin}^{-} \mathrm{CD} 122^{+} \mathrm{NK} 1.1^{-} \mathrm{NKp} 46^{-}$. They develop into immature NK cells (iNKs) that become $\mathrm{NK} 1.1^{+}$while only mature NK cells (mNKs) are NK1.1 ${ }^{+} \mathrm{NKp} 46^{+}(22)$. As the Cre recombinase expression in Ncr1Cre mice is driven by the NKp46 promoter, Cre-mediated deletion is restricted to $\mathrm{mNK}$ cells. We observed a significant decrease of percentage and total numbers of bone marrow NK cells in Jak1 ${ }^{f / f l} N$ crlCre mice (Figure 1A). The Lin $^{-}$CD $122^{+}$NK cells in Jak $1^{f l / f l}$ NcrlCre mice showed enriched percentages of NK cell precursors (NKPs) and immature NK cells, while $\mathrm{mNKs}$ were significantly reduced in the bone marrow in line with a developmental block at the iNK cell stage preventing progression to $\mathrm{mNK}$ cell stage (Figure 1B). Deletion of one allele of Jak1 led to intermediate numbers of bone marrow NK cells (Figure 1A). Consistently, NK cell development showed an intermediate phenotype suggesting a Jakl gene-dosage effect on NK cell development (Figure 1B).

The block in development of bone marrow NK cells translated into drastically reduced numbers of NK cells in the periphery. Loss of JAK1 led to an almost complete deficiency of splenic and blood NK cells (Figures 1C-E). In line with a Jak1 gene dosage effect, Jak $1^{f l /+}$ NcrlCre mice displayed reduced NK cell percentages and total numbers to $50 \%$ compared to wild-type littermates in the spleen and blood (Figures 1C-E). Deletion of the JAK1 downstream effector and transcription factor STAT5 in $\mathrm{NKp} 46^{+}$cells also leads to a reduction of mature NK cells (18). A direct comparison of $\mathrm{Jak} 1^{f l / f l} \mathrm{Ncr} 1 \mathrm{Cre}$ and Stat $5^{f l / f l} \mathrm{Ncr} 1 \mathrm{Cre}$ mice revealed that deletion of JAK1 provoked an even more pronounced NK cell deficiency in spleen and blood than deletion of STAT5 (Figures S1A,B).

Liver $\mathrm{NKp} 46^{+}$innate lymphocytes comprise two groups of distinct lineages (23). Conventional NK cells (cNK) are characterized by expression of CD49b and circulate freely whereas liver resident type 1 innate lymphocytes (ILC1) are characterized by the expression of CD49a and are restricted to the liver (24). Similarly to spleen and blood, liver cNKs and tissue resident ILC1s were almost completely ablated upon loss of JAK1 (Figure 1F). Again the deletion of one allele of Jak1 resulted in an intermediate abundance of liver innate lymphocytes (Figure 1F). In summary, these findings led us to conclude that JAK1 expression in $\mathrm{NKp} 46^{+}$cells is indispensable for $\mathrm{NK}$ cell development and maintenance in peripheral organs in a dose-dependent manner.

\section{JAK1 Is Crucial for NK Cell Maturation}

In the periphery NK cells undergo maturation steps which are characterized by sequential expression of CD27 and CD11b surface markers (22). One allele of Jak1 was sufficient to drive NK cell maturation as we did not detect any differences in percentage of cells in each maturation stage between $J a k 1^{W T}$ and Jak1 ${ }^{f l /+}$ Ncr1Cre cells (Figures 2A,B). The remaining Jak $1^{f l / f l}$ NcrlCre NK cells showed an increase in the immature population $\left(\mathrm{CD} 27^{+} \mathrm{CD} 11 \mathrm{~b}^{-}\right)$and a decrease in the mature $\mathrm{CD} 27^{-} \mathrm{CD}_{11 b^{+}}$population (Figures $2 \mathrm{~A}, \mathbf{B}$ and Figure S1C). This result suggests that the remaining cells might just have lost

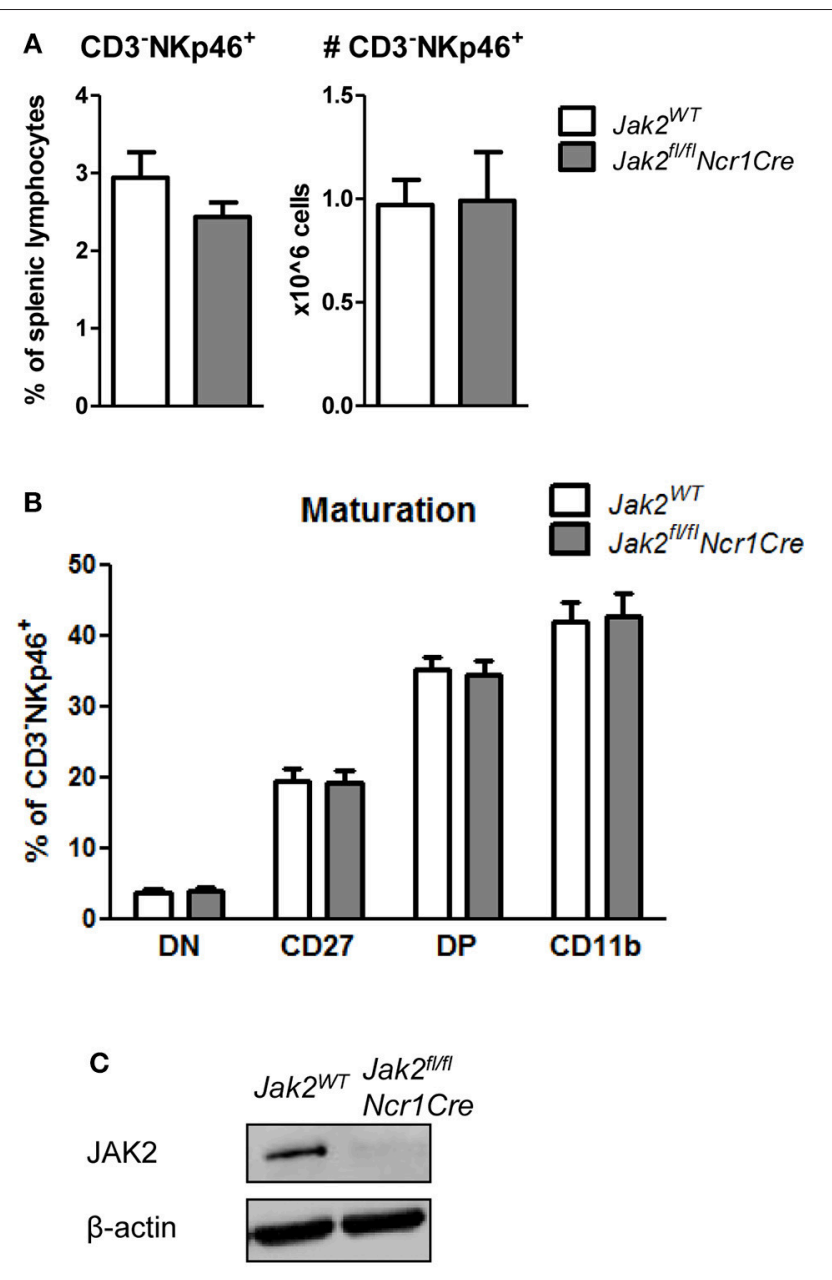

FIGURE 3 | JAK2 is dispensable for NK cell survival and maturation. (A) Frequency (left panel) and total numbers (right panel) of CD3-NKp46 ${ }^{+} \mathrm{NK}$ cells in the spleens of Jak2 ${ }^{W T}$ and Jak $2^{f l / f l}$ Ncr1Cre mice were assessed by flow cytometry. (B) Splenic CD3 ${ }^{-} \mathrm{NKp} 46^{+}$NK cells were analyzed for expression of CD27 and CD11b by flow cytometry. Frequency of cells in each maturation stage is show: DN (CD27- CD11b $\left.{ }^{-}\right), \mathrm{CD} 27\left(\mathrm{CD} 27^{+} \mathrm{CD} 11 \mathrm{~b}^{-}\right)$, DP $\left(\mathrm{CD} 27^{+} \mathrm{CD} 11 \mathrm{~b}^{+}\right), \mathrm{CD} 11 \mathrm{~b}\left(\mathrm{CD} 27^{-} \mathrm{CD} 11 \mathrm{~b}^{+}\right)$. (C) The expression of JAK2 and $\beta$-actin was analyzed by Western blot in NK cells upon 6 days of expansion in IL-2. Scans of full blots are available in Supplementary Material. (A,B) Bar graphs represent mean \pm SEM of 2-3 independent experiments; $n=5-12$.

JAK1 and have not received sufficient IL-15 signaling to fully mature. Indeed, the remaining Jak $1^{f l / f l} N c r 1 C r e$ NK cells showed reduced phosphorylation of STAT5 ex vivo upon short-term stimulation with IL-15 (Figure 2C). NK cell activity is controlled by a balance between activating and inhibitory receptors. Deletion of neither one nor both alleles of Jak1 had an effect on the percentage of NK cells expressing following activating and inhibitory receptors: KLRG1, NKG2D, NKG2A/C/E, and Ly49A (Figure 2D). The most prominent difference was a slight decrease of Ly49G2 ${ }^{+} \mathrm{NK}$ cells and an increase in DNAM- $1^{+} \mathrm{NK}$ cells in the Jak1 $1^{f l / f l} \mathrm{Ncr} 1 \mathrm{Cre}$ mice (Figure 2D). Similarly, no gross differences were detected in the expression level (MFI) of each receptor, besides an increase in the MFI of DNAM1 and Ly49G2 


\section{A}

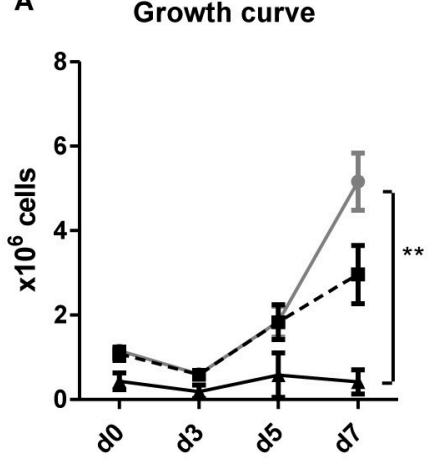

C

YAC-1

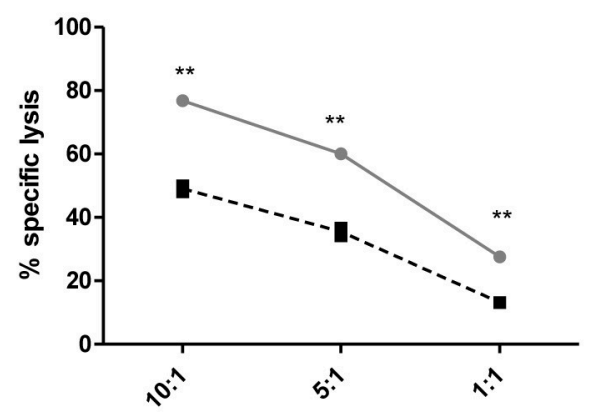

B

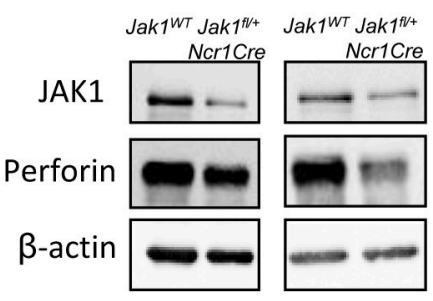

RMA-Rae1

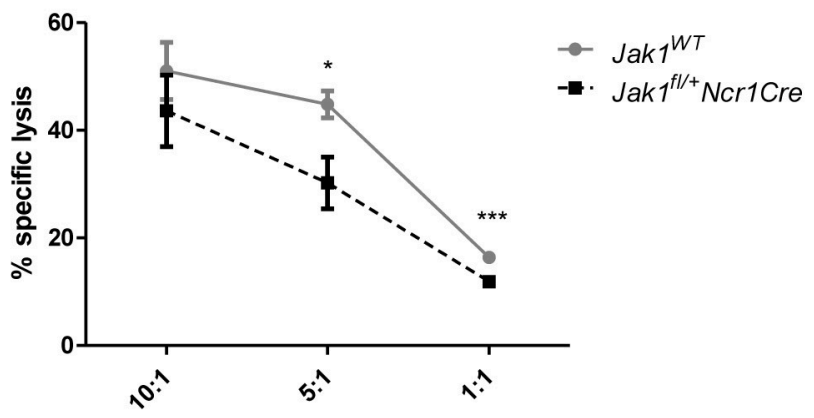

FIGURE 4 | Loss of one allele of Jak1 impairs NK cell activity. (A) NK cells were MACS-purified from spleens and cultured in IL-2 for 7 days. The numbers of living

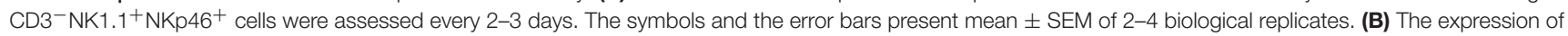
JAK1, Perforin and $\beta$-actin was analyzed in expanded NK cells from two independent experiments by Western blot. Scans of full blots are available in

Supplementary Material. (C) Expanded NK cells from Jak1 ${ }^{W T}$ and Jak $1^{f l /}+\mathrm{Ncr} 1 \mathrm{Cr}$ mice were mixed with CFSE-stained YAC-1 (left panel) or RMA-Rae1 (right panel) target cells at indicated effector target ratios. The specific lysis was assessed by flow cytometry. For YAC-1 a representative graph of one of two independent experiments is shown. The symbols and the error bars present mean \pm SEM of 2 technical replicates. For RMA-Rae1 a mean out of two independent experiments is shown. The symbols and the error bars present mean \pm SEM of $2-3$ biological replicates. ${ }^{*} p<0.05,{ }^{\star \star} p<0.01,{ }^{\star \star \star} p<0.001$.

(Figure S1D). Besides its role as an activating receptor, DNAM1 expression marks a developmental step; DNAM-1 ${ }^{+}$cells give rise to DNAM-1 ${ }^{-}$cells (25). We reasoned that the changes in DNAM $-1^{+}$reflect the maturation block of Jakl ${ }^{f l / f l} \mathrm{Ncr} 1 \mathrm{Cre}$ NK cells.

\section{JAK2 Is Dispensable for NK Cell Survival and Maturation}

So far we showed that NK cell-intrinsic JAK1 deletion leads to NK cell deficiency. To elucidate if JAK2 impacts on NK cell survival and maturation, we analyzed splenic NK cells in Jak2 $2^{f l / f l} \mathrm{Ncr} 1 \mathrm{Cre}$ mice and their wild-type littermates. We failed to detect any impact of JAK2 deletion on the frequency or total numbers of $\mathrm{CD}^{-} \mathrm{NKp}^{-} 6^{+}$cells in the spleen (Figure 3A). Furthermore, $J a k 2^{f l / f l}$ Ncr1Cre mice showed normal NK cell maturation, as similar percentages of $\mathrm{CD}_{2} 7^{-} \mathrm{CD} 11 \mathrm{~b}^{+}$cells were detected in both genotypes (Figure 3B). As the deletion of JAK2 protein in NK cells was very efficient (Figure 3C), these data unequivocally define that unlike JAK1, NK cell-intrinsic JAK2 is dispensable for NK cell survival and maturation.

\section{Loss of One Allele of Jak1 Impairs NK Cell Functionality}

To get further insights into how JAK1 regulates NK cell functionality, we analyzed the growth of MACS-purified splenic NK cells from Jak1 $1^{W T}$, Jak1 $1^{f l+}$ Ncr1Cre, and Jak1 $1^{f l / f l} \mathrm{Ncr} 1 \mathrm{Cre}$. JAK1-deficient NK cells did not expand, which shows that even under a very high dose of IL-2 other JAKs cannot compensate for the loss of JAK1 (Figure 4A). The loss of one allele of Jak1 resulted in a minor growth deficiency (Figure 4A). Western blot analysis confirmed the reduced JAK1 protein expression in expanded Jak1 $1^{f l+}$ NcrlCre NK cells which was paralleled by reduced levels of perforin (Figure 4B). In line, Jakl $1^{f l /+} \mathrm{Ncr} 1 \mathrm{Cre}$ NK cells displayed an impaired cytotoxic activity against target cell lines YAC-1 and RMA-Rae1 (Figure 4C). These results prove that JAK1 is not only indispensable for maintaining NK cells in periphery, but also contributes to their cytotoxic activity.

\section{NK Cell Depletion Induced by Loss of One Allele of Jak1 Impairs Tumor Surveillance}

NK cells are crucial for the early recognition and elimination of transformed cells. To investigate whether NK cell reduction 
A

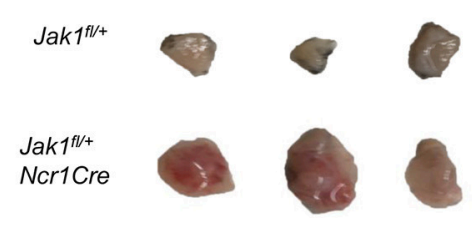

C

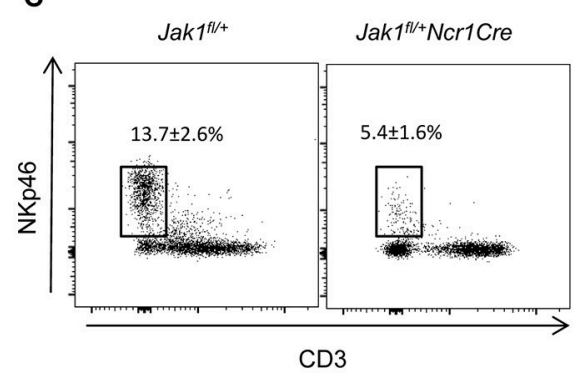

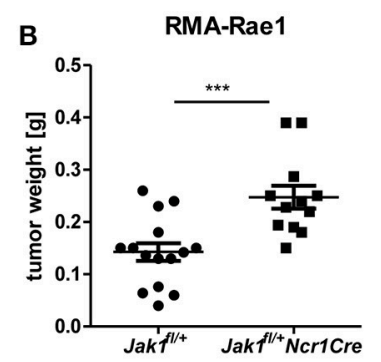

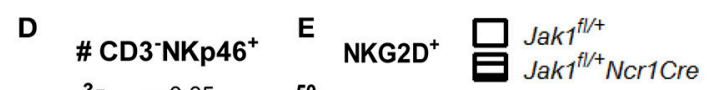
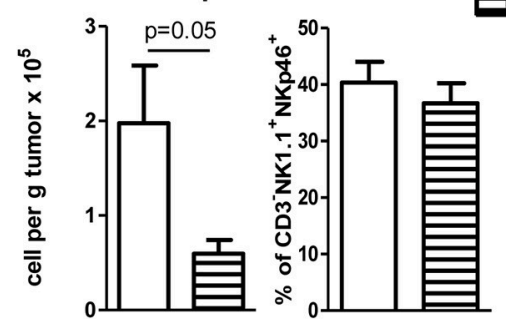

FIGURE 5 | Loss of one allele of Jak1 impairs tumor surveillance. (A,B) Jak $1^{f l / t}$ and Jak $1^{f l /}+$ Ncr 1 Cre mice were injected s.C. with $10^{6}$ RMA-Rae 1 cells and after 10 days the tumor weight was assessed. Shown are (A) representative tumor pictures and (B) dot plots with horizontal lines representing mean tumor weights \pm SEM from 2 independent experiments; $n=6-8$ (C,D) Tumor infiltrating NK cells were analyzed by flow cytometry. (C) Representative plots of tumor infiltrating

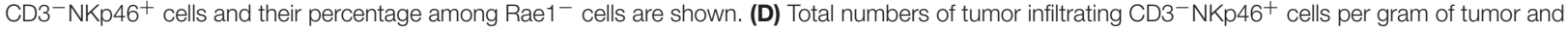
(E) percentages of NKG2D ${ }^{+}$NK cells are presented as bar graphs showing mean \pm SEM from one experiment $n=4-5$. ${ }^{* \star *} p<0.001$.

and their impaired functionality in $J a k 1^{f l+}$ NcrlCre mice results in increased susceptibility to tumor growth and is not compensated by other means, we made use of RMA-Rael lymphoma cells. This cell line is a tool to study NK celldependent tumor surveillance in a robust and efficient way (20). We subcutaneously transplanted RMA-Rael lymphoma cells into both flanks of Jak $f^{f l+}$ and Jak $f^{f l+}$ Ncr1Cre mice. Lacking one allele of Jak1 impaired the ability of NK cells to control the tumor growth as illustrated by increased tumor size (Figure 5A) and tumor weight in Jak $\mathrm{f}^{f l+} \mathrm{Ncr} 1 \mathrm{Cre}$ (Figure 5B). In line, these tumors showed significantly reduced NK cell infiltration (Figures 5C,D). We failed to detect any difference in the frequency of $\mathrm{NKG}_{2} \mathrm{D}^{+}$tumor infiltrating $\mathrm{NK}$ cells (Figure 5E), which are crucial for the recognition of RMA-Rae1 tumors. In summary, our results show the reduced NK cell numbers combined with an impaired NK cell functionality in Jak $1^{f l+}$ NcrlCre mice are sufficient to significantly impair tumor growth control in vivo.

\section{DISCUSSION}

Dysregulation of the JAK-STAT signaling pathway is tightly associated with cancer development as well as immune disorders (7). The first JAK-linked disease discovered was the severe combined immune deficiency (SCID) which was characterized by NK cell abnormalities caused by LOF JAK3 mutations $(26,27)$. We here show that deletion of JAK1 in NKp $46^{+}$cells leads to innate immune deficiency with loss of NK and ILC1 cells in peripheral organs, whereas JAK2 is redundant for NK cell survival and maturation.

Our previous work uncovered that loss of STAT5 in NK cells leads to severe reduction of NK cell numbers in peripheral organs (18). The loss of NK cells in Stat $5^{f l / f l} \mathrm{Ncr} 1 \mathrm{Cre}$ mice was rescued by enforced expression of the pro-survival molecule BCL2 (28). This study defined STAT5 as a crucial survival factor for NK cells. STAT5B-deficient mice largely lack NK cells (29), in line with the fact that STAT5 signals downstream of cytokines that are vital for NK cell biology, such as IL-2 or IL-15 (30). IL-15 is crucial for NK cell development and survival as $I l 15^{-/-}$mice are largely devoid of peripheral NK cells (31). IL-15 signals via a receptor complex of $\gamma c$ receptor chain, IL-2R $\beta$, and IL-15r $\alpha$ (32). Knockout mice of each receptor chain prove an absolutely critical role for signals sent downstream of IL-15 for NK cell development $(33,34)$. To date, the contribution of $\gamma c$-associated JAK3 to NK cell development has been well-established. Mice with JAK3 deficiency show a similar SCID phenotype as observed in human patients and NK cell development is blocked at the pre-NK progenitor stage $(35,36)$. Now we place previously underappreciated JAK1 as a crucial part of the IL-15/STAT5 axis in NK cells. Jak $1^{f / / f l} \mathrm{Ncr} 1 \mathrm{Cre}$ NK cells show a developmental block at the iNK cell stage and an almost complete loss of NK cells in peripheral organs.

Interestingly, the consequences of JAK1 deletion for NK cells exceed the effects of STAT5-deficiency. Impairment of combined STAT3 and STAT5 activation may underlie the more pronounced loss of NK cells, as STAT3 has been shown to induce expression of the crucial NK cell survival gene Mcl1 $(37,38)$. Alternatively, 
JAK1 and STAT5 may have different half-lives which account for different frequencies of "just deleters"-NK cells which have just lost the gene but still carry the protein, that may explain differences.

The gene-dosage effect of JAK1-deficiency is reflected in NK cell numbers, while loss of one allele of JAK1 is dispensable for NK cell maturation. This suggests that activated STAT5 is rate limiting for NK cell survival but not maturation. Deletion of one allele of Jakl is also sufficient to significantly impair tumor surveillance based on decreased numbers of NK cells combined with a diminished functionality of the remaining NK cells. In accordance with our data, NK cell-specific deletion of the STAT5 target gene $\mathrm{Mcl} 1 \mathrm{l}$ leads to severe NK cell deficiency which causes a significant increase in metastatic burden (38).

JAKs may exhibit redundant functions and compensate for each other. Downstream of interferon $\gamma$ receptor JAK1 can partially compensate for loss of JAK2 kinase activity (39). JAK2 has also been shown to phosphorylate STAT5 downstream of IL15 during in vitro differentiation of NK cells (16). On the other hand, constitutively active JAK2 can only modestly compensate for the loss of JAK1 in stem cells suggesting a non-redundant role of JAK1 and 2 in HSCs. In line, we observe that in the absence of JAK1, JAK2 fails to compensate in activating STAT5, even under high dose of IL-2 in vitro, to allow NK cell survival. It remains to be elucidated whether compensatory effects are achievable by expressing a constitutively active form of JAK2. More importantly, we show that loss of JAK2 in NKp46 ${ }^{+}$cells is dispensable for NK cell survival. JAK2-deficient NK cells are fully mature, proving that NK cell-intrinsic JAK2 is not driving NK cell maturation. This also indicates that the impaired NK cell maturation in Jak $2^{f l / f l} \mathrm{M} x 1 \mathrm{Cre}$ mice (14) is most likely caused by NK cell-extrinsic functions of JAK2. One might speculate that the constitutive deletion of JAK2 alters the cytokine milieu.

\section{REFERENCES}

1. Abel AM, Yang C, Thakar MS, Malarkannan S. Natural killer cells: development, maturation, and clinical utilization. Front Immunol. (2018) 9:1869. doi: 10.3389/fimmu.2018.01869

2. Orange JS. Natural killer cell deficiency. J Allergy Clin Immunol. (2013) 132:515-25. doi: 10.1016/j.jaci.2013.07.020

3. O'Shea JJ, Plenge R. JAK and STAT signaling molecules in immunoregulation and immune-mediated disease. Immunity (2012) 36:542-50. doi: 10.1016/j.immuni.2012.03.014

4. Stark GR, Darnell JE. The JAK-STAT pathway at twenty. Immunity (2012) 36:503-14. doi: 10.1016/j.immuni.2012.03.013

5. Shuai K, Liu B. Regulation of JAK-STAT signalling in the immune system. Nat Rev Immunol. (2003) 3:900-11. doi: 10.1038/nri1226

6. O'Shea JJ, Holland SM, Staudt LM. JAKs and STATs in immunity, immunodeficiency, and cancer. $N$ Engl J Med. (2013) 368:161-70. doi: 10.1056/NEJMra1202117

7. Hammarén HM, Virtanen AT, Raivola J, Silvennoinen O. The regulation of JAKs in cytokine signaling and its breakdown in disease. Cytokine (2018). doi: 10.1016/j.cyto.2018.03.041. [Epub ahead of print].

8. Lorenzini T, Dotta L, Giacomelli M, Vairo D, Badolato R. STAT mutations as program switchers: turning primary immunodeficiencies into autoimmune diseases. J Leukoc Biol. (2017) 101:29-38. doi: 10.1189/jlb.5RI0516-237RR

9. Haan C, Rolvering C, Raulf F, Kapp M, Drückes P, Thoma G, et al. Jak1 has a dominant role over Jak3 in signal transduction through
Our data provide novel insights into results obtained upon JAK1/JAK2 inhibitor treatment. Inhibition of JAK1/JAK2 reduces NK cell numbers and maturation $(14,15)$, which according to our data is clearly the effect of inhibiting JAK1 rather than JAK2. We thus propose that the development of JAK2specific inhibitors may be advantageous as they would leave NK cell-mediated tumor surveillance intact. This might be of particular relevance in the case of JAK2-driven leukemia such as $\mathrm{JAK} 2^{\mathrm{V} 617 \mathrm{~F}}$-induced myeloproliferative neoplasms.

\section{AUTHOR CONTRIBUTIONS}

AW-S, KK, DP, and NL performed experiments. AW-S analyzed the data. VS, GS, and AD provided the resources. AW-S and VS wrote the manuscript.

\section{FUNDING}

The work was supported by the Austrian Science Fund FWF grant SFB-F61 to VS.

\section{ACKNOWLEDGMENTS}

We thank Philipp Jodl for great technical support and Manuela Kindl for maintaining and monitoring the mice. We are grateful to Andrea Hölbl-Kovacic, Dagmar Gotthardt, and Eva Maria Putz for critical feedback on the manuscript.

\section{SUPPLEMENTARY MATERIAL}

The Supplementary Material for this article can be found online at: https://www.frontiersin.org/articles/10.3389/fimmu. 2018.03108/full\#supplementary-material

yc-containing cytokine receptors. Chem Biol. (2011) 18:314-23. doi: 10.1016/j.chembiol.2011

10. Eletto D, Burns SO, Angulo I, Plagnol V, Gilmour KC, Henriquez F, et al. Biallelic JAK1 mutations in immunodeficient patient with mycobacterial infection. Nat Commun. (2016) 7:13992. doi: 10.1038/ncomms13992

11. Rodig SJ, Meraz MA, White JM, Lampe PA, Riley JK, Arthur CD, et al. Disruption of the Jak1 gene demonstrates obligatory and nonredundant roles of the Jaks in cytokine-induced biologic responses. Cell (1998) 93:373-83.

12. Kleppe M, Spitzer MH, Li S, Hill CE, Dong L, Papalexi E, et al. Jak1 Integrates cytokine sensing to regulate hematopoietic stem cell function and stress hematopoiesis. Cell Stem Cell (2018) 22:277. doi: 10.1016/j.stem.2017

13. Schwartz DM, Kanno Y, Villarino A, Ward M, Gadina M, O'Shea JJ. JAK inhibition as a therapeutic strategy for immune and inflammatory diseases. Nat Rev Drug Discov. (2017) 16:843-62. doi: 10.1038/nrd.2017.201

14. Bottos A, Gotthardt D, Gill JW, Gattelli A, Frei A, Tzankov A, et al. Decreased NK-cell tumour immunosurveillance consequent to JAK inhibition enhances metastasis in breast cancer models. Nat Commun. (2016) 7:12258. doi: $10.1038 /$ ncomms 12258

15. Schönberg K, Rudolph J, Vonnahme M, Parampalli Yajnanarayana S, Cornez I, Hejazi $M$, et al. JAK inhibition impairs NK cell function in myeloproliferative neoplasms. Cancer Res. (2015) 75:2187-99. doi: 10.1158/0008-5472.CAN-14-3198

16. Kim WS, Kim MJ, Kim DO, Byun J-E, Huy H, Song HY, et al. Suppressor of cytokine signaling 2 negatively regulates NK cell differentiation by inhibiting JAK2 activity. Sci Rep. (2017) 7:46153. doi: 10.1038/srep46153 
17. Krempler A, Qi Y, Triplett AA, Zhu J, Rui H, Wagner K-U. Generation of a conditional knockout allele for the Janus kinase 2 (Jak2) gene in mice. Genesis (2004) 40:52-7. doi: 10.1002/gene.20063

18. Eckelhart E, Warsch W, Zebedin E, Simma O, Stoiber D, Kolbe T, et al. A novel Ncr1 -Cre mouse reveals the essential role of STAT5 for NK-cell survival and development. Blood (2011) 117:1565-74. doi: 10.1182/blood-2010-06-291633

19. Cui Y, Riedlinger G, Miyoshi K, Tang W, Li C, Deng C, et al. Inactivation of Stat5 in mouse mammary epithelium during pregnancy reveals distinct functions in cell proliferation, survival, and differentiation. Mol Cell Biol. (2004) 18:8037-47. doi: 10.1128/MCB.24.18.8037-8047.2004

20. Cerwenka A, Baron JL, Lanier LL. Ectopic expression of retinoic acid early inducible-1 gene (RAE-1) permits natural killer cell-mediated rejection of a MHC class I-bearing tumor in vivo. Proc Natl Acad Sci USA. (2001) 98:11521-6. doi: 10.1073/pnas.201238598

21. Putz E, Gotthardt D, Hoermann G, Csiszar A, Wirth S, Berger A, et al. CDK8-mediated STAT1-S727 phosphorylation restrains NK cell cytotoxicity and tumor surveillance. Cell Rep. (2013) 4:437-44. doi: 10.1016/j.celrep.2013.07.012

22. Geiger TL, Sun JC. Development and maturation of natural killer cells. Curr Opin Immunol. (2016) 39:82-9. doi: 10.1016/j.coi.2016.01.007

23. Sojka DK, Plougastel-Douglas B, Yang L, Pak-Wittel M, Artyomov MN, Ivanova $\mathrm{Y}$, et al. Tissue-resident natural killer (NK) cells are cell lineages distinct from thymic and conventional splenic NK cells. Elife (2014) 3:e01659. doi: 10.7554/eLife.01659

24. Peng H, Jiang X, Chen Y, Sojka DK, Wei H, Gao X, et al. Liver-resident NK cells confer adaptive immunity in skin-contact inflammation. J Clin Invest. (2013) 123:1444-56. doi: 10.1172/JCI66381

25. Martinet L, Ferrari De Andrade L, Guillerey C, Lee JS, Liu J, Souza-FonsecaGuimaraes F, et al. DNAM-1 expression marks an alternative program of NK cell maturation. Cell Rep. (2015) 11:85-97. doi: 10.1016/j.celrep.2015. 03.006

26. Macchi P, Villa A, Giliani S, Sacco MG, Frattini A, Porta F, et al. Mutations of Jak-3 gene in patients with autosomal severe combined immune deficiency (SCID). Nature (1995) 377(6544):65-8.

27. Russell SM, Tayebi N, Nakajima H, Riedy MC, Roberts JL, Aman MJ, et al. Mutation of Jak3 in a patient with SCID: essential role of Jak3 in lymphoid development. Science (1995) 270:797-800.

28. Gotthardt D, Putz EM, Grundschober E, Prchal-Murphy M, Straka E, Kudweis $\mathrm{P}$, et al. STAT5 is a key regulator in NK cells and acts as molecular switch from tumor surveillance to tumor promotion. Cancer Discov. (2016) 4:414-29. doi: 10.1158/2159-8290.CD-15-0732

29. Villarino A V., Sciumè G, Davis FP, Iwata S, Zitti B, Robinson GW, et al. Subset- and tissue-defined STAT5 thresholds control homeostasis and function of innate lymphoid cells. J Exp Med. (2017) 214:2999-3014. doi: $10.1084 /$ jem. 20150907
30. Marçais A, Viel S, Grau M, Henry T, Marvel J, Walzer T. Regulation of mouse NK cell development and function by cytokines. Front Immunol. (2013) 4:450. doi: 10.3389/fimmu.2013.00450

31. Kennedy MK, Glaccum M, Brown SN, Butz EA, Viney JL, Embers $\mathrm{M}$, et al. Reversible defects in natural killer and memory CD8 T cell lineages in interleukin 15-deficient mice. J Exp Med. (2000) 191:771-80. doi: 10.1084/jem.191.5.771

32. Ikemizu S, Chirifu M, Davis SJ. IL-2 and IL-15 signaling complexes: different but the same. Nat Immunol. (2012) 13:1141-2. doi: 10.1038/ni.2472

33. Williams NS, Klem J, Puzanov IJ, Sivakumar PV, Schatzle JD, Bennett M, et al. Natural killer cell differentiation: insights from knockout and transgenic mouse models and in vitro systems. Immunol Rev. (1998) 165:47-61.

34. Lodolce JP, Boone DL, Chai S, Swain RE, Dassopoulos T, Trettin S, et al. IL-15 receptor maintains lymphoid homeostasis by supporting lymphocyte homing and proliferation. Immunity (1998) 9:669-76.

35. Park SY, Saijo K, Takahashi T, Osawa M, Arase H, Hirayama N, et al. Developmental defects of lymphoid cells in Jak3 kinase-deficient mice. Immunity (1995) 3:771-82.

36. Robinette ML, Cella M, Telliez JB, Ulland TK, Barrow AD, Capuder K, et al. Jak3 deficiency blocks innate lymphoid cell development. Mucosal Immunol. (2017) 1:50-60. doi: 10.1038/mi.2017.38

37. Abdulghani J, Allen JE, Dicker DT, Liu YY, Goldenberg D, Smith CD, et al. Sorafenib sensitizes solid tumors to Apo2L/TRAIL and Apo2L/TRAIL receptor agonist antibodies by the Jak2-Stat3-Mcl1 axis. PLoS ONE (2013) 8:e75414. doi: 10.1371/journal.pone.0075414

38. Sathe P, Delconte RB, Souza-Fonseca-Guimaraes F, Seillet C, Chopin $M$, Vandenberg $C J$, et al. Innate immunodeficiency following genetic ablation of Mcl1 in natural killer cells. Nat Commun. (2014) 5:4539. doi: $10.1038 /$ ncomms5539

39. Keil E, Finkenstädt D, Wufka C, Trilling M, Liebfried P, Strobl B, et al. Important scaffold function of the Janus kinase 2 uncovered by a novel mouse model harboring a Jak2 activation-loop mutation. Blood (2014) 123:520-9. doi: 10.1182/blood-2013-03-492157

Conflict of Interest Statement: The authors declare that the research was conducted in the absence of any commercial or financial relationships that could be construed as a potential conflict of interest.

Copyright (C) 2019 Witalisz-Siepracka, Klein, Prinz, Leidenfrost, Schabbauer, Dohnal and Sexl. This is an open-access article distributed under the terms of the Creative Commons Attribution License (CC BY). The use, distribution or reproduction in other forums is permitted, provided the original author(s) and the copyright owner(s) are credited and that the original publication in this journal is cited, in accordance with accepted academic practice. No use, distribution or reproduction is permitted which does not comply with these terms. 